\title{
Nonclassicality in phase-number uncertainty relations
}

\author{
Paloma Matía-Hernando and Alfredo Luis* \\ Departamento de Óptica, Facultad de Ciencias Físicas, Universidad Complutense, 28040 Madrid, Spain
}

(Received 25 September 2011; published 12 December 2011)

\begin{abstract}
We show that there are nonclassical states with lesser joint fluctuations of phase and number than any classical state. This is rather paradoxical since one would expect classical coherent states to be always of minimum uncertainty. The same result is obtained when we replace phase by a phase-dependent field quadrature. Number and phase uncertainties are assessed using variance and Holevo relation.
\end{abstract}

DOI: 10.1103/PhysRevA.84.063829

PACS number(s): 42.50.Ct, 42.50.Lc, 42.50.Dv

\section{INTRODUCTION}

Uncertainty relations are usually presented as a typical quantum feature. More specifically, the quantum theory imposes a lower bound to the joint uncertainty of noncommuting observables, such as position and momentum in mechanics, or phase and number in optics. The mere existence of joint fluctuations is not a quantum signature, and in classical optics fluctuations are the rule, as demonstrated by the degrees of polarization and coherence [1]. Naturally, classical optics admits fields with no fluctuations at all.

Within quantum optics it is customary to distinguish between classical states (light states compatible with classical optics) and nonclassical states [1-3]. In this work we investigate whether there is any difference between these categories regarding uncertainty relations. This is whether joint uncertainty may show any evidence of nonclassical behavior of the electromagnetic field, revealed by field states with lesser joint uncertainty than any classical state.

The answer to this query depends on the variables and uncertainty measures considered. For Cartesian unbounded variables, such as position and momentum or field quadratures, the minimum uncertainty states include the classical coherent states [2], so no nonclassical behavior is disclosed by uncertainty relations. In this work we show that this is not always the case when other field variables are considered. We demonstrate that there are nonclassical states with lesser joint phase-number uncertainty than any classical state. Number and phase uncertainties are assessed using variance and Holevo relation [4-7].

This result is somewhat paradoxical since according standard physical intuition one might expect that the minimum uncertainty states should always include classical states. Seemingly, classical states should be the closest to classical optics, where there are fields with no fluctuations at all. Moreover, classical coherent states have the largest degrees of coherence [1] and polarization [8] allowed by the quantum theory, which agrees with the idea of minimum uncertainty. This counterintuitive behavior parallels recent results concerning complementarity [9]. The approach followed in this work (nonclassicality from the point of view of joint phase-number uncertainty) is complementary to previous approaches where

*alluis@ fis.ucm.es; [http://www.ucm.es/info/gioq] nonclassicality was addressed exclusively in terms of the individual number [1,2] or phase [10] statistics.

In Sec. II we will recall the quantum description of phase and number and their fluctuations, as well as typical classical and nonclassical states associated with these variables. In Sec. III we examine whether there are nonclassical states with lesser phase-number joint uncertainty than any classical state. Finally, in Sec. IV we show that equivalent results are obtained when we replace phase by a more experimentally accessible phase-dependent field quadrature.

\section{PHASE, NUMBER, CLASSICAL, AND NONCLASSICAL STATES}

For completeness, in this section we recall basic facts to be used later about phase, number, classical, and nonclassical states.

\section{A. Phase and number}

Throughout this paper we will focus on a single-mode field described by the complex amplitude operator $a$ with $\left[a, a^{\dagger}\right]=$ 1. The number operator is well defined as $a^{\dagger} a$, which we will refer to as $n$ when there is no risk of confusion. The number states $a^{\dagger} a|n\rangle=n|n\rangle$ define the number statistics as

$$
p(n)=\langle n|\rho| n\rangle,
$$

where $\rho$ is any density matrix.

On the other hand, the description of quantum phase is more troublesome $[7,11]$. We will consider that the phase variable is represented in the quantum domain by the phase states $|\phi\rangle$ defined as the eigenstates of the Susskind-Glogower operator $E$ with unit-modulus eigenvalue [12]

$$
E|\phi\rangle=e^{i \phi}|\phi\rangle, \quad|\phi\rangle=\frac{1}{\sqrt{2 \pi}} \sum_{n=0}^{\infty} e^{i n \phi}|n\rangle,
$$

and

$$
E=\int_{\phi_{0}}^{\phi_{0}+2 \pi} d \phi|\phi\rangle e^{i \phi}\left\langle\phi\left|=\sum_{n=1}^{\infty}\right| n-1\right\rangle\langle n|,
$$

where $\phi_{0}$ is any phase. These phase states define suitable phase statistics $p(\phi)$ for every state $\rho$ as

$$
p(\phi)=\langle\phi|\rho| \phi\rangle .
$$




\section{Holevo relation}

A suitable phase-number uncertainty relation can be derived from standard procedures starting from the cosine and sine operators

$$
C=\frac{1}{2}\left(E+E^{\dagger}\right), \quad S=\frac{1}{2 i}\left(E-E^{\dagger}\right),
$$

satisfying the commutation relations

$$
\left[a^{\dagger} a, S\right]=i C, \quad\left[a^{\dagger} a, C\right]=-i S,
$$

that lead to

$$
(\Delta n)^{2}(\Delta C)^{2} \geqslant \frac{1}{4}\langle S\rangle^{2}, \quad(\Delta n)^{2}(\Delta S)^{2} \geqslant \frac{1}{4}\langle C\rangle^{2} .
$$

Taking into account that

$$
C^{2}+S^{2}=I-\frac{|0\rangle\langle 0|}{2}, \quad|\langle E\rangle|^{2}=\langle C\rangle^{2}+\langle S\rangle^{2},
$$

we get the uncertainty relation for $\langle E\rangle \neq 0$

$$
H=(\Delta n)^{2}\left(\frac{1-\frac{\langle\mid 0\rangle\langle 0 \mid\rangle}{2}}{|\langle E\rangle|^{2}}-1\right) \geqslant \frac{1}{4},
$$

that henceforth we will refer to as the Holevo relation [4-7], while for $\langle E\rangle=0$ we get

$$
(\Delta n)^{2}\left(1-\frac{\langle\mid 0\rangle\langle 0 \mid\rangle}{2}\right) \geqslant 0 .
$$

\section{B. Classical states}

A key point of this approach is a clear definition of what can be understood by classical states within the quantum theory. We will consider the most standard definition, although this is not unique [13], which is that classical states are the ones with Glauber-Sudarshan $P$ function compatible with classical optics [i.e., $P(\alpha)$ nonnegative and no more singular than a $\delta$ function] [1,2],

$$
\rho=\int d^{2} \alpha P(\alpha)|\alpha\rangle\langle\alpha|,
$$

with $|\alpha\rangle$ being the coherent states (eigenstates of the complex amplitude operator $a|\alpha\rangle=\alpha|\alpha\rangle)$.

The prototypical example (and the only pure case) is the coherent states $|\alpha\rangle$, which in the number basis read

$$
|\alpha\rangle=e^{-|\alpha|^{2} / 2} \sum_{n=0}^{\infty} \frac{\alpha^{n}}{\sqrt{n !}}|n\rangle .
$$

This is the Poissonian number distribution,

$$
p(n)=|\langle n \mid \alpha\rangle|^{2}=e^{-|\alpha|^{2}} \frac{|\alpha|^{2 n}}{n !},
$$

with $\bar{n}=\left\langle\alpha\left|a^{\dagger} a\right| \alpha\right\rangle=|\alpha|^{2}$ and

$$
\langle\mid 0\rangle\langle 0 \mid\rangle=e^{-\bar{n}}, \quad(\Delta n)^{2}=\overline{n^{2}}-\bar{n}^{2}=\bar{n} .
$$

Unfortunately, there is no simple expression for the phase distribution $p(\phi)$ or $\langle E\rangle$ :

$$
\langle E\rangle=e^{i \arg \alpha} e^{-\bar{n}} \sum_{n=1}^{\infty} \frac{\bar{n}^{n-\frac{1}{2}}}{\sqrt{n !(n-1) !}} .
$$

Nevertheless, for large enough photon numbers $\bar{n} \gg 1$ the number distribution admits a Gaussian approximation assuming that $n$ is a continuous variable, which leads to

$$
|\langle E\rangle|^{2} \simeq 1-\frac{1}{4(\Delta n)^{2}} .
$$

\section{Nonclassical states}

Let us briefly present the nonclassical states that will be invoked in this work.

\section{Squeezed states}

Squeezed states are characterized by Gaussian quadrature statistics having quadrature fluctuations below the level established by coherent states,

$$
\Delta X=\frac{e^{r}}{2}, \quad \Delta Y=\frac{e^{-r}}{2},
$$

where $r$ is a real parameter, being $r=0$ for coherent states, and

$$
X=\frac{1}{2}\left(a+a^{\dagger}\right), \quad Y=\frac{i}{2}\left(a^{\dagger}-a\right) .
$$

A suitable expression in the number basis (for $\bar{Y}=0$ for simplicity) is $|\xi\rangle=\sum_{n=0}^{\infty} c_{n}|n\rangle$, with

$$
\begin{aligned}
c_{n}= & \frac{(-\tanh r)^{n / 2}}{\sqrt{2^{n} n ! \cosh r}} \exp \left[-\frac{R^{2}}{2}(1-\tanh r)\right] \\
& \times H_{n}\left[\frac{i R}{\sqrt{2}}\left(\sqrt{\tanh r}-\frac{1}{\sqrt{\tanh r}}\right)\right],
\end{aligned}
$$

where $H_{n}$ are the Hermite polynomials and $\bar{X}=R$. The mean number of photons is

$$
\bar{n}=\left\langle\xi\left|a^{\dagger} a\right| \xi\right\rangle=R^{2}+\sinh ^{2} r
$$

while the uncertainty is

$$
(\Delta n)^{2}=R^{2} \mathrm{e}^{2 r}+\frac{1}{2} \sinh ^{2}(2 r) .
$$

For large $\bar{n}$ and parameters $r$ close to zero, $r \simeq 0$, the continuous-number and Gaussian approximation of the number and phase statistics will hold.

\section{Coherent phase states}

The coherent phase states are the normalizable eigenstates of $E$ [12]:

$$
E|\zeta\rangle=\zeta|\zeta\rangle, \quad|\zeta\rangle=\sqrt{1-|\zeta|^{2}} \sum_{n=0}^{\infty} \zeta^{n}|n\rangle
$$

with $|\zeta|<1$,

$$
\bar{n}=\frac{|\zeta|^{2}}{1-|\zeta|^{2}}, \quad|\langle E\rangle|^{2}=\frac{\bar{n}}{1+\bar{n}},
$$

and

$$
\langle\mid 0\rangle\langle 0 \mid\rangle=\frac{1}{1+\bar{n}}, \quad(\Delta n)^{2}=\bar{n}(1+\bar{n}) .
$$




\section{Phase-number intermediate states}

Joint uncertainty relations of two observables are often minimized by states with properties somewhat intermediate between the two observables. So it might be interesting to consider the eigenstates of the following combinations of number and phase observables [5], $n+i \lambda E^{\dagger}$ and $n+i \lambda E$, where $\lambda$ is a real parameter.

In the case of

$$
\left(n+i \lambda E^{\dagger}\right)|\psi\rangle=\mu|\psi\rangle,
$$

there are solutions only for $\mu=0$, being

$$
|\psi\rangle=\frac{1}{\sqrt{I_{0}(2 \lambda)}} \sum_{n=0}^{\infty} \frac{(-i \lambda)^{n}}{n !}|n\rangle,
$$

with

$$
\bar{n}=\lambda \frac{I_{1}(2 \lambda)}{I_{0}(2 \lambda)}, \quad(\Delta n)^{2}=\lambda^{2}-\bar{n}^{2},
$$

where $I_{1,0}$ are the corresponding modified Bessel functions and

$$
\langle\mid 0\rangle\langle 0 \mid\rangle=\frac{1}{I_{0}(2 \lambda)}, \quad\langle E\rangle=-i \frac{\bar{n}}{\lambda} .
$$

On the other hand, in the case of

$$
(n+i \lambda E)|\psi\rangle=\mu|\psi\rangle,
$$

there are solutions only if $\mu$ is a natural number $\mu=N$ leading to

$$
|\psi\rangle=\mathcal{N} \sum_{n=0}^{N} \frac{N !}{(N-n) !(i \lambda)^{n}}|n\rangle,
$$

where $\mathcal{N}$ is a normalization constant.

\section{NONCLASSICALITY IN THE HOLEVO RELATION}

The border between classical and nonclassical behaviors is fixed by the minimum value of the joint uncertainty for classical states. We may conjecture that for each $\bar{n}$, classical mixed states will have larger joint fluctuations than pure classical states, so that the minimum joint uncertainty will be achieved by the corresponding pure coherent state $|\alpha\rangle$ with $|\alpha|^{2}=\bar{n}$. In Appendix we provide an explicit demonstration of this conjecture for number and quadrature variances in the general case, as well as for the phase uncertainty for small photon numbers.

A numerical evaluation of the left-hand side $H$ of the Holevo relation (9) for classical pure coherent states is presented in Fig. 1 (throughout this work plotted quantities are dimensionless). This figure provides a classical-quantum frontier in the sense that only nonclassical states can lie between the curve $H_{\text {class }}$ and the horizontal dashed line at 1/4, which represent the left- and right-hand sides of Eq. (9), respectively. It can be appreciated that the classical coherent states tend to saturate the uncertainty relation as $\bar{n}$ increases.

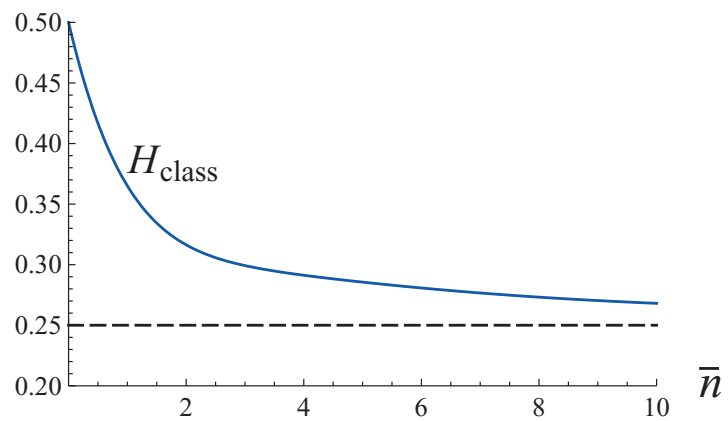

FIG. 1. (Color online) Numerical evaluation of the left-hand side $H$ of the Holevo relation (9) for coherent states (solid line) as a function of the mean number of photons $\bar{n}$. The dashed line corresponds to the lower limit $1 / 4$. Throughout the work plotted quantities are dimensionless.

\section{A. Limit of large photon numbers}

For large mean photon numbers $\bar{n}$ the continuous number and Gaussian approximations hold for coherent states. For $\bar{n} \gg 1$ we have after Eq. (14)

$$
\langle\mid 0\rangle\langle 0 \mid\rangle=e^{-\bar{n}} \simeq 0,
$$

and after Eq. (16) we get

$$
\frac{1}{|\langle E\rangle|^{2}}-1 \simeq \frac{1}{4(\Delta n)^{2}},
$$

with $(\Delta n)^{2}=\bar{n} \gg 1$, so that

$$
\frac{1-\frac{\langle\mid 0\rangle\langle 0 \mid\rangle}{2}}{|\langle E\rangle|^{2}}-1 \simeq \frac{1}{4(\Delta n)^{2}} .
$$

This means that the classical pure coherent states become minimum joint-uncertainty states of the Holevo relation (9) $H \simeq 1 / 4$. Thus, for large photon numbers (after Fig. 1 say, for example, $\bar{n}>10$ ) it is not practical to look for nonclassical states with joint uncertainty smaller than classical since the difference with the coherent-state uncertainty will be minimal and hardly noticeable. We conclude that the phase-number uncertainty relation cannot display nonclassical properties in this limit so we must turn our attention to the case of small photon numbers.

\section{B. Small photon numbers}

According to the results of the case of large photon numbers, if we intend to disclose nonclassical behavior $H<H_{\text {class }}$ we must focus on the case of fixed and small $\bar{n}$ so that the continuous number and Gaussian approximations do not hold. More specifically, according to Fig. 1, we may find clearly noticeable nonclassical behavior in joint phase-number uncertainty for typically $\bar{n} \simeq 1$. Let us consider some examples.

\section{Squeezed states}

For definiteness we consider states with $\bar{Y}=0$. In this case we have found nonclassical uncertainty in the case of very slight negative squeezing, $r<0$ with $|r| \simeq 0$, which after 


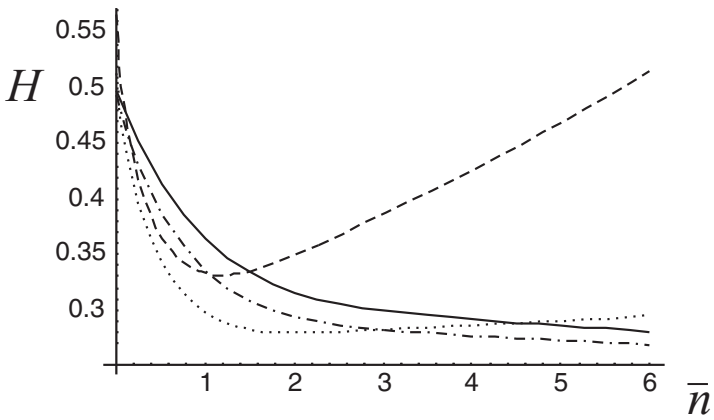

FIG. 2. Numerical evaluation of the left-hand side $H$ of the Holevo relation (9) as a function of $\bar{n}$ for different squeezed states as functions of the mean number of photons $\bar{n}$. Expressing $R$ as $R=\eta \sqrt{\bar{n}}$ we have plotted the cases $\eta=0.95$ (dashed line), $\eta=0.98$ (dotted line), and $\eta=0.999$ (dash-dotted line). The solid line is the classical coherent state $\eta=1$.

Eq. (17) for $\bar{Y}=0$ implies reduced amplitude fluctuations. This is illustrated in Fig. 2, which shows the left-hand side of the Holevo relation (9) as a function of $\bar{n}$ for different amounts of negative squeezing. The best results are obtained for $|r| \simeq 0$; this is for $R \simeq \sqrt{\bar{n}}$, which are very close to classical coherent states.

In Fig. 3 we have represented the left-hand side $H$ of the Holevo relation (9) relative to $H_{\text {class }}(\bar{n})$ for fixed $\bar{n}$ as a function of $R / R_{\max }$, where $R_{\max }=\sqrt{\bar{n}}$, for different values of the mean number of photons $\bar{n}$. For increasing $R$ (this is decreasing squeezing), first the nonclassicality increases but then it decreases as the state tends to be coherent as $R \rightarrow \sqrt{\bar{n}}$. Moreover, for increasing $\bar{n}$ the possibility of nonclassicality decreases and holds for lesser squeezing (i.e., $R$ closer to $\left.R_{\max }\right)$.

This behavior admits a simple description. For states close to the vacuum and phase squeezing the phase distribution acquires two opposite peaks increasing both phase and number uncertainties [14]. Thus, optimum results are obtained when we move away from the vacuum as much as possible (this is increasing $R$ ), with slight amplitude squeezing (this is $r<0$ ). Although this squeezing increases phase uncertainty it is compensated by a more effective decrease of the number uncertainty.

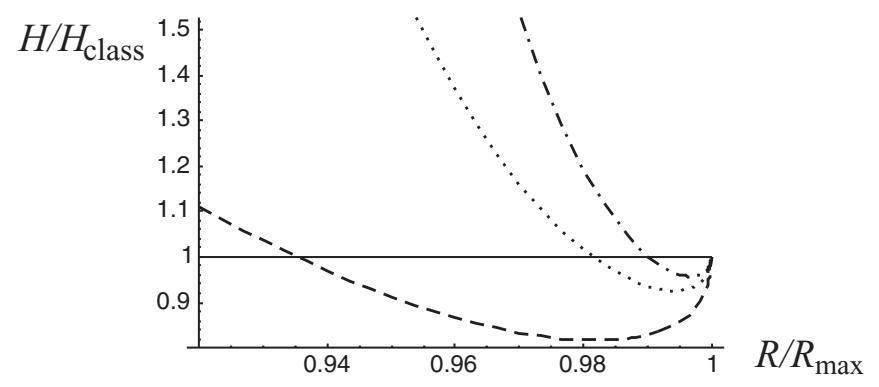

FIG. 3. Left-hand side $H$ of the Holevo relation (9) relative to $H_{\text {class }}(\bar{n})$ a function of $R / R_{\max }$ with $R_{\max }=\sqrt{\bar{n}}$ for different values of the mean number of photons, $\bar{n}=1$ (dashed line), $\bar{n}=5$ (dotted line), and $\bar{n}=10$ (dash-dotted line).

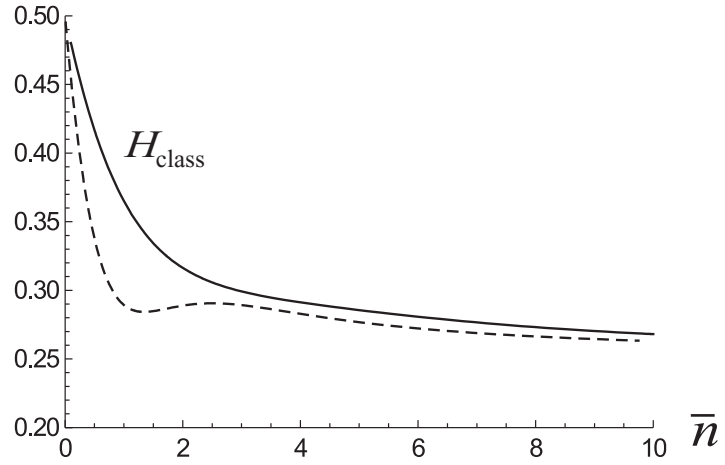

FIG. 4. Left-hand side $H$ of the Holevo relation (9) for the eigenstates of $n+i \lambda E^{\dagger}$ (dashed line) and for coherent states (solid line) as functions of the mean number of photons $\bar{n}$.

\section{Coherent phase states}

After Eq. (24) there is a simple analytical expression for the left-hand side $H$ of the Holevo relation (9):

$$
H=\frac{1+\bar{n}}{2} \text {. }
$$

This means that these states are always in the classical side of the border in Fig. 2. This is consistent with the result for squeezed states, in the sense that nonclassicality holds by reducing number fluctuations, instead of by reducing phase fluctuations.

\section{Intermediate states}

In Fig. 4 we have represented the left-hand side $H$ of the Holevo relation (9) as a function of $\bar{n}$ for the eigenstates of $n+i \lambda E^{\dagger}$ in Eq. (27), showing nonclassical behavior for all $\bar{n}$. On the other hand, in Fig. 5 we have represented $H$ as a function of $\bar{n}$ for the eigenstates of $n+i \lambda E$ in Eq. (30), showing nonclassical behavior for some values of $\bar{n}$ for all values of $N$ considered, especially for small $N$.

\section{Nonclassicality around the vacuum}

Let us address an analytical approximate analysis of the nonclassical behavior $H<H_{\text {class }}$ for $\bar{n} \ll 1$. Since we are interested in very low photon number states we

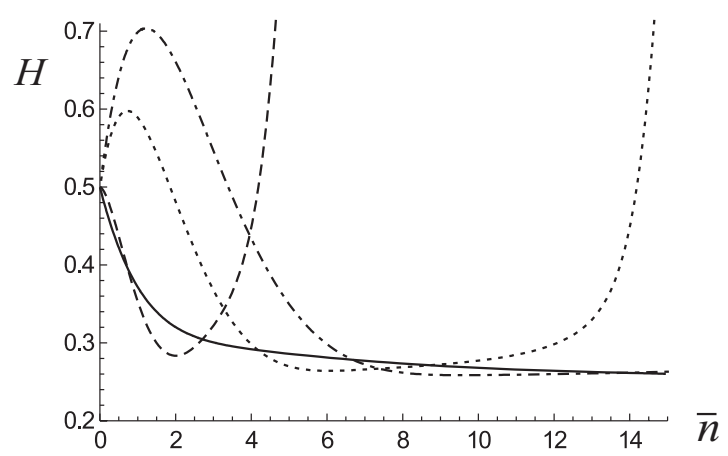

FIG. 5. Left-hand side $H$ of the Holevo relation (9) for the eigenstates of $n+i \lambda E$ for different values of $N, N=5$ (dashed line), $N=15$ (dotted line), $N=25$ (dash-dotted line), and for coherent states (solid line), as functions of the mean number of photons $\bar{n}$. 
may approximate the state in the number basis in the form

$$
|\psi\rangle \approx \sqrt{\mathcal{N}}\left(|0\rangle+\alpha|1\rangle+\gamma \frac{\alpha^{2}}{\sqrt{2}}|2\rangle+\cdots\right),
$$

where $\mathcal{N}$ is a normalization factor,

$$
\mathcal{N} \simeq \frac{1}{1+\alpha^{2}+\frac{\gamma^{2} \alpha^{4}}{2}} \simeq 1-\alpha^{2}+\left(1-\frac{\gamma^{2}}{2}\right) \alpha^{4},
$$

while $\alpha$ and $\gamma$ are state parameters assumed real for simplicity. From a generic expression in the photon-number basis $|\psi\rangle=\sum_{n} c_{n}|n\rangle$ these parameters are given by $\alpha=$ $c_{1} / c_{0}$ and $\gamma=\sqrt{2} c_{0} c_{2} / c_{1}^{2}$. Classical coherent states have $\gamma=1$.

Within this approximation the left-hand side $H$ of the Holevo relation (9) is, retaining terms up to $\alpha^{2}$,

$$
H \simeq \frac{1}{2}\left[1+\left(2 \gamma^{2}-\sqrt{2} \gamma-1\right) \bar{n}\right],
$$

where $\bar{n} \simeq \alpha^{2}$. For coherent states $\gamma=1$ we get

$$
H_{\mathrm{class}} \simeq \frac{1}{2}[1+(1-\sqrt{2}) \bar{n}],
$$

so that nonclassicality $H<H_{\text {class }}$ implies that

$$
2 \gamma^{2}-\sqrt{2} \gamma-2+\sqrt{2}<0
$$

which holds for $1>\gamma>1 / \sqrt{2}-1 \simeq-0.3$.

This agrees with the preceding numerical results. On the one hand, since within this approximation $(\Delta n)^{2} \simeq \bar{n}-$ $2\left(1-\gamma^{2}\right) \bar{n}^{2}$ we get that $H<H_{\text {class }}$ requires sub-Poissonian statistics $(\Delta n)^{2}<\bar{n}$, so that amplitude squeezing is preferred in front of phase squeezing. Moreover, for squeezed states $\gamma=$ $1+\tanh r /\left[R^{2}(1-\tanh r)^{2}\right]$ that for small $r$ scales as $\gamma \simeq$ $1+r / R^{2}$, this confirms that nonclassical behavior requires $r<0$. For the intermediate states we have $\gamma=1 / \sqrt{2}$ for the eigenstates of $n+i \lambda E^{\dagger}$ so that they all display nonclassical uncertainty, while $\gamma=\sqrt{2}(1-1 / N)$ for the eigenstates of $n+i \lambda E$, so that within this approximation of $\bar{n} \ll 1$ there is nonclassicality only for $N \leqslant 3$. This agrees with the results in Figs. 4 and 5. Finally, $\gamma=\sqrt{2}$ for the coherent phase states so they are always in the classical region.

\section{NONCLASSICALITY IN THE NUMBER-QUADRATURE UNCERTAINTY RELATION}

A drawback of the quantum phase is that it has no simple measuring procedure [15]. Because of this we can complete the analysis exploring a related practical scheme, where phase is replaced by a field quadrature, say $X$. This is a phase-sensitive observable that can be easily measured in practice via homodyne detection [2] (another alternative can be found in Ref. [16]). In this case, the commutation relation

$$
\left[a^{\dagger} a, X\right]=-i Y
$$

leads to

$$
(\Delta n)^{2}(\Delta X)^{2} \geqslant \frac{1}{4} \bar{Y}^{2} \text {. }
$$

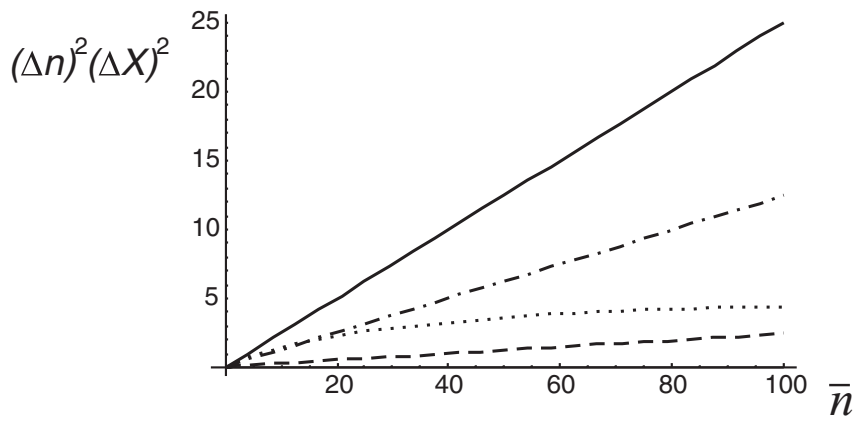

FIG. 6. Plot of $(\Delta n)^{2}(\Delta X)^{2}$ as a function of $\bar{n}$ for different squeezed states $R=\eta \sqrt{\bar{n}}$ with $\eta=0.9$ (dashed line), $\eta=0.999$ (dotted line), and $\eta=0.1$ (dash-dotted line); the solid line is the classical coherent state.

By computing $\Delta n \Delta X$ for classical pure coherent states we readily obtain the following lower bound valid for every classical state with mean number of photons $\bar{n}$ :

$$
(\Delta n)^{2}(\Delta X)^{2} \geqslant \frac{\bar{n}}{4} .
$$

Next we investigate whether there are nonclassical states with smaller $\Delta n \Delta X$ for fixed $\bar{n}>0$.

A first clear example is number states with

$$
\Delta n=0, \quad(\Delta X)^{2}=\frac{1}{4}+\frac{\bar{n}}{2},
$$

so that $\Delta n \Delta X$ is clearly below the classical limit.

This can also be the case of squeezed states. Again, the most favorable case is amplitude squeezing $r<0$, since this may decrease the fluctuations of both $n$ and $X$ simultaneously. In Fig. 6 we have represented $(\Delta n)^{2}(\Delta X)^{2}$ as a function of $\bar{n}$ for several distributions of the photons between the coherent and the squeezed parts (given by $R$ and $r$, respectively), along with the classical limit $\bar{n} / 4$. It can be appreciated that the nonclassical behavior is larger for small squeezing ( $R$ close to its maximum value $\sqrt{\bar{n}}$ ) and is maintained for arbitrary large photon numbers.

In Fig. 7 we have represented the relative joint uncertainty $(\Delta n)^{2}(\Delta X)^{2} /(\bar{n} / 4)$ as a function of $R / R_{\max }$, where $R_{\max }=$ $\sqrt{\bar{n}}$, for different values of the mean number of photons

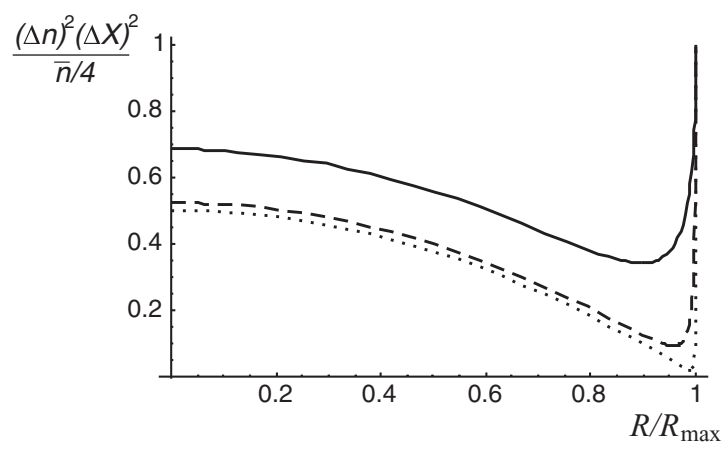

FIG. 7. Plot of $(\Delta n)^{2}(\Delta X)^{2} /(\bar{n} / 4)$ as a function of $R / R_{\max }$ where $R_{\max }=\sqrt{\bar{n}}$ for different values of the mean number of photons $\bar{n}=1$ (solid line), $\bar{n}=10$ (dashed line), and $\bar{n}=100$ (dotted line). 


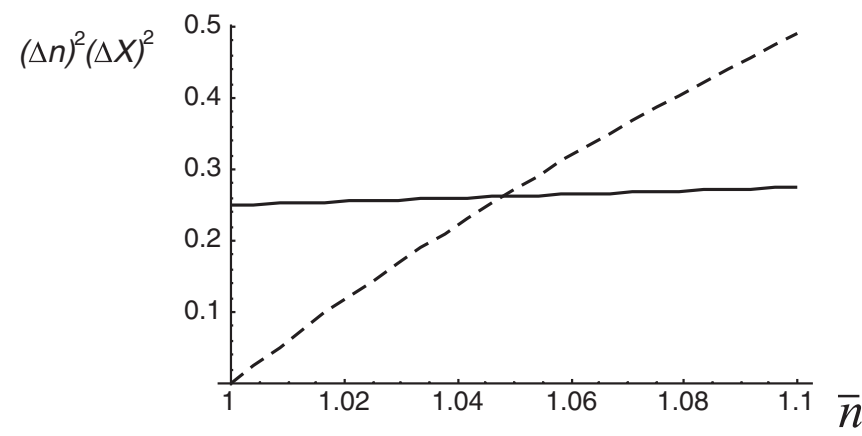

FIG. 8. Plot of $(\Delta n)^{2}(\Delta X)^{2}$ (dashed line) and $\bar{n} / 4$ (solid line) as functions of $\bar{n}$ for crescent states with $N=1$.

$\bar{n}$. This shows that there is nonclassical behavior for all $R$. The nonclassicality is clearer for very small squeezing (this is $R$ close to $R_{\max }$ ). The optimum squeezing is smaller for larger $\bar{n}$.

We can also consider number-quadrature intermediate states as the eigenstates of $a^{\dagger} a-i \lambda\left(a^{\dagger}+a\right)$, the so-called crescent states [17,18], leading to [17]

$$
(\Delta n)^{2}(\Delta X)^{2}=\frac{\lambda^{2}}{4}\left[1+\frac{L_{N}^{1}\left(-4 \lambda^{2}\right)}{L_{N}\left(-4 \lambda^{2}\right)}\right]
$$

where $N$ is any natural number, $L_{N}^{k}$ are generalized Laguerre polynomials, and the mean number of photons is $\bar{n}=N+\lambda^{2}$. We have found nonclassical behavior for $N \geqslant 1$ only for values of $\lambda$ very close to zero (this is just for intermediate states very close to number states) as illustrated in Fig. 8, where nonclassical behavior only takes place for $\lambda \leqslant 0.2$.

Finally, for coherent phase states we have found numerically that the product $\Delta n \Delta X$ is always above the classical lower bound, so they display no nonclassical behavior.

\section{CONCLUSIONS}

We have shown that there are nonclassical states with lesser joint phase-number and quadrature-number uncertainty than any classical state. This result is somewhat paradoxical since one might expect classical states to be closer to classical physics, where there is room for states with no fluctuations at all. Moreover, we have shown that this nonclassical behavior is favored by amplitude squeezing instead of phase squeezing.

\section{ACKNOWLEDGMENTS}

A. L. acknowledges support from Project No. FIS200801267 of the Spanish Dirección General de Investigación del Ministerio de Ciencia e Innovación, and from Project QUITEMAD S2009-ESP-1594 of the Consejería de Educación de la Comunidad de Madrid.

\section{APPENDIX: PURE VS MIXED CLASSICAL STATES IN NUMBER VARIANCE, QUADRATURE VARIANCE, AND HOLEVO PHASE UNCERTAINTY}

In this Appendix we demonstrate that for the same mean number of photons $\bar{n}$ the pure coherent states $|\alpha\rangle$ have lesser uncertainty than any other mixed classical state $\rho$,

$$
\rho=\int d^{2} \alpha P(\alpha)|\alpha\rangle\langle\alpha|
$$

with $P(\alpha) \geqslant 0$. We do this for number variance, quadrature variance, and the Holevo phase uncertainty (in this last case just for very small photon numbers).

\section{Number variance}

For number variance we have

$$
(\Delta n)^{2}=\left\langle a^{\dagger} a^{\dagger} a a\right\rangle+\bar{n}-\bar{n}^{2},
$$

so that using Eq. (A1) we get

$$
(\Delta n)_{\rho}^{2}=\int d^{2} \beta P(\beta)|\beta|^{4}+\bar{n}-\bar{n}^{2} \geqslant \bar{n}=(\Delta n)_{|\alpha\rangle}^{2},
$$

where $|\alpha\rangle$ is a coherent state with $|\alpha|=\sqrt{\bar{n}}$, where we have used that for classical distributions $P(\beta)$ it holds that

$$
\int d^{2} \beta P(\beta)|\beta|^{4} \geqslant\left[\int d^{2} \beta P(\beta)|\beta|^{2}\right]^{2}=\bar{n}^{2} .
$$

This is $\bar{z}^{2} \geqslant \bar{z}^{2}$ for any classical random variable $z$. The equality holds exclusively when $\rho$ is a coherent state $|\alpha\rangle$, so that mixed classical states have larger number variance than the pure coherent states with the same $\bar{n}$.

\section{Quadrature variance}

For the quadrature operator $X=\left(a^{\dagger}+a\right) / 2$ and using Eq. (A1) we get for classical states with the same $\bar{n}$

$$
(\Delta X)_{\rho}^{2}=\int d^{2} \beta P(\beta)(x-\bar{x})^{2}+\frac{1}{4} \geqslant \frac{1}{4}=(\Delta X)_{|\alpha\rangle}^{2},
$$

where $x$ is the real part of $\beta$ and $|\alpha\rangle$ is any coherent state. The equality holds exclusively when $\rho$ is a coherent state $|\alpha\rangle$, so that mixed classical states have larger quadrature variance than the pure coherent states.

\section{Phase uncertainty}

In this case we simplify the analysis considering that we are very close to the vacuum, so that the coherent states in Eq. (A1) can be expressed in the number basis as

$$
|\alpha\rangle \simeq\left(1-|\alpha|^{2} / 2\right)\left(|0\rangle+\alpha|1\rangle+\frac{\alpha^{2}}{\sqrt{2}}|2\rangle+\cdots\right),
$$

so that the phase uncertainty is

$$
\left(\frac{1-\frac{\langle\mid 0\rangle\langle 0 \mid\rangle}{2}}{|\langle E\rangle|^{2}}-1\right) \simeq \frac{1+\bar{n}}{2\left|\int d^{2} \beta P(\beta) \beta\right|^{2}}-1 \geqslant \frac{1+\bar{n}}{2 \bar{n}}-1,
$$


where the last expression on the right-hand side is the phase uncertainty in a coherent state $|\alpha\rangle$ with $|\alpha|=\sqrt{\bar{n}}$, and we have used that for classical states

$$
\begin{aligned}
\left|\int d^{2} \beta P(\beta) \beta\right|^{2} & \leqslant\left(\int d^{2} \beta P(\beta)|\beta|\right)^{2} \\
& \leqslant \int d^{2} \beta P(\beta)|\beta|^{2}=\bar{n} .
\end{aligned}
$$

The equality holds exclusively when the statistical operator $\rho$ is a coherent state $|\alpha\rangle$, so that mixed classical states have larger phase uncertainty than the pure coherent states, for the same small enough $\bar{n}$.

Actually, it can be seen that within this approximation the inequality (A7) holds for every state since

$$
\left|\int d^{2} \beta P(\beta) \beta\right|^{2}=\bar{X}^{2}+\bar{Y}^{2} \leqslant \overline{X^{2}}+\overline{Y^{2}}-\frac{1}{2}=\bar{n},
$$

where the inequality holds because for all states $(\Delta X)^{2}+$ $(\Delta Y)^{2} \geqslant 1 / 2$, the equality being reached exclusively by coherent states. This again points out that the reduction of the joint number-phase uncertainty requires, essentially, a reduction of number fluctuations.
[1] L. Mandel and E. Wolf, Optical Coherence and Quantum Optics (Cambridge University Press, Cambridge, England, 1995).

[2] M. O. Scully and M. S. Zubairy, Quantum Optics (Cambridge University Press, Cambridge, England, 1997).

[3] V. V. Dodonov, J. Opt. B 4, R1 (2002).

[4] A. S. Holevo, in Quantum Probability and Applications to the Quantum Theory of Irreversible Processes, Springer Lecture Notes in Mathematics Vol. 1055, edited by L. Accardi, A. Frigerio, and V. Gorini (Springer, Berlin, 1984), p. 153.

[5] A. Lukš and V. Peřinová, Phys. Rev. A 45, 6710 (1992); A. Lukš, V. Peřinová, and J. Křepelka, ibid. 46, 489 (1992).

[6] J. M. Lévy-Leblond, Ann. Phys. (NY) 101, 319 (1976).

[7] P. Carruthers and M. M. Nieto, Rev. Mod. Phys. 40, 411 (1968).

[8] A. Luis, Phys. Rev. A 66, 013806 (2002).

[9] A. Luis, Phys. Rev. A 84, 012106 (2011).

[10] D. Martín and A. Luis, Phys. Rev. A 82, 033829 (2010).

[11] D. T. Pegg and S. M. Barnett, J. Mod. Opt. 44, 225 (1997); R. Lynch, Phys. Rep. 256, 367 (1995); V. Peřinová, A. Lukš, and J. Peřina, Phase in Optics (World Scientific, Singapore, 1998).
[12] E. C. Lerner, H. W. Huang, and G. E. Walters, J. Math. Phys. 11, 1679 (1970); A. Vourdas, Phys. Rev. A 45, 1943 (1992); E. C. G. Sudarshan, Int. J. Theor. Phys. 32, 1069 (1993); A. Vourdas, Phys. Scr. T 48, 84 (1993); A. Vourdas, C. Brif, and A. Mann, J. Phys. A 29, 5887 (1996); A. Vourdas, ibid. 39, R65 (2006); C. C. Gerry and T. Bui, Phys. Rev. A 80, 033831 (2009).

[13] J. A. Vaccaro, Opt. Commun. 113, 421 (1995); Phys. Rev. A 52, 3474 (1995); J. A. Vaccaro and D. T. Pegg, ibid. 41, 5156 (1990); L. M. Johansen, Phys. Lett. A 329, 184 (2004); J. Opt. B 6, L21 (2004); L. M. Johansen and A. Luis, Phys. Rev. A 70, 052115 (2004); A. Luis, ibid. 73, 063806 (2006).

[14] W. Schleich, R. J. Horowicz, and S. Varro, Phys. Rev. A 40, 7405 (1989).

[15] D. T. Smithey, M. Beck, J. Cooper, and M. G. Raymer, Phys. Rev. A 48, 3159 (1993); T. Opatrný, M. Dakna, and D.-G. Welsch, ibid. 57, 2129 (1998).

[16] I. Urizar-Lanz and G. Tóth, Phys. Rev. A 81, 052108 (2010).

[17] Z. Hradil, Phys. Rev. A 44, 792 (1991).

[18] A. Lukš, V. Peřinová, and J. Křepelka, J. Mod. Opt. 41, 2325 (1994). 